\title{
The comparative effects of intravenous iron on oxidative stress and inflammation in patients with chronic kidney disease and iron deficiency: a randomized controlled pilot study
}

\author{
Xenophon Kassianides ${ }^{1}$, Andrew Gordon ${ }^{2}$, Roger Sturmey ${ }^{2}$, Sunil Bhandari ${ }^{1}$ \\ ${ }^{1}$ Academic Renal Research Department, Hull University Teaching Hospitals NHS Trust and Hull York Medical School, Hull Royal Infirmary, \\ Hull, UK \\ ${ }^{2}$ Centre for Atherothrombosis and Metabolic Disease, Hull York Medical School, University of Hull, Hull, UK
}

Background: Concerns exist regarding the pro-oxidant and inflammatory potential of intravenous (IV) iron due to labile plasma iron (LPI) generation. This IRON-CKD trial compared the effects of different IV irons on oxidative stress and inflammation.

Methods: In this randomized open-label explorative single-center study in the United Kingdom, non-dialysis-dependent chronic kidney disease (CKD) patients with iron deficiency were randomized (1:1:1:1) to receive a single infusion of $200 \mathrm{mg}$ iron dextran, or 200 mg iron sucrose (IS), or 200 mg or 1,000 mg ferric derisomaltose (FDI) and were followed up for 3 months. The primary outcomes measured were induction of oxidative stress and inflammation. Secondarily, efficacy, vascular function, quality of life, and safety were monitored.

Results: Forty patients were enrolled. No significant rise in oxidative stress existed, regardless of preparation or dose. There was a significant rise in LPI with 1,000 mg FDI at 2 hours that normalized within a week, not impacting oxidative stress or inflammation. A delayed rise in C-reactive protein was noted with IS. High-dose FDI produced a sustained serum ferritin increase (mean \pm standard error of the mean of predose: $69.1 \pm 18.4 \mu \mathrm{g} / \mathrm{L}, 3$ months: $271.0 \pm 83.3 \mu \mathrm{g} / \mathrm{L} ; \mathrm{p}=0.007)$. Hemoglobin remained stable throughout. No adverse drug reactions were recorded during the study.

Conclusion: A single dose of IV iron in CKD patients does not trigger oxidative stress or inflammation biomarkers. Third-generation IV irons have a reassuring safety profile, and high-dose FDI produced a sustained serum ferritin rise and more efficient iron repletion, with no significant pro-oxidant or inflammatory signals when compared to a lower dose and other IV irons.

Keywords: Chronic kidney disease, Ferric derisomaltose, Intravenous iron, Iron deficiency, Oxidative stress

Received: July 7, 2020; Revised: July 30, 2020; Accepted: August 25, 2020

Editor: Eun Hui Bae, Chonnam National University, Gwangju, Republic of Korea

Correspondence: Xenophon Kassianides

Hull University Teaching Hospitals NHS Trust and Hull York Medical School, Hull Royal Infirmary, Anlaby Road, HU3 2JZ Hull, UK. Email: xenophon. kassianides@hey.nhs.uk

ORCID: https://orcid.org/0000-0002-1452-7757

Copyright (c) 2021 by The Korean Society of Nephrology

(a) This is an Open Access article distributed under the terms of the Creative Commons Attribution Non-Commercial and No Derivatives License (http:// creativecommons.org/licenses/by-nc-nd/4.0/) which permits unrestricted non-commercial use, distribution of the material without any modifications, and reproduction in any medium, provided the original works properly cited. 


\section{Introduction}

Iron deficiency anemia is a global condition co-existing with other medical comorbidities including chronic kidney disease (CKD), heart failure, inflammatory bowel disease, and malignancy $[1,2]$. It is associated with prognostic and financial implications and reduced quality of life [3,4].

Despite the broad availability of oral iron, issues remain with compliance and side effects [4]. Consequently, intravenous (IV) iron replacement has gained popularity and is incorporated in current clinical guidelines $[5,6]$. Several IV iron preparations are available including second-generation (e.g., iron sucrose [IS], low molecular weight iron dextran [ID]), and third-generation (e.g., ferric derisomaltose [FDI]) products.

Concerns exist regarding the risks of oxidative stress with parenteral iron administration [7-9]. Previous evidence suggests that the degree of oxidative stress caused by iron supplementation is relative to the labile plasma iron (LPI) released $[10,11]$. LPI can be involved in redox cycling and has a major role in the initiation and propagation of lipid peroxidation [12]. Works by Zager et al. $[8,13,14]$ have indicated both a dose-related and a preparation-dependent association between iron and toxicity, especially with second-generation IV iron products (IVIPs). The amount of LPI released is related to the structural stability of the ironcarbohydrate complex; third-generation IV irons represent more tightly bound compounds than second-generation products [15]. This may explain why less stable IVIPs (e.g., IS) are linked to endothelial and renal dysfunction and inflammation [16-18].

This explorative study was designed to primarily assess the effect of different IV iron compounds and dosages on oxidative stress and inflammation in patients with nondialysis-dependent CKD and absolute or functional iron deficiency.

Additionally, the effects of these IV iron treatments on hematinic profile and hemoglobin concentration, arterial stiffness, endothelial function, quality of life, and safety outcomes were investigated. We hypothesized that treatment with IV irons would primarily lead to variable effects on biomarkers of inflammation and oxidative stress and secondarily may result in differences in efficacy markers.

\section{Methods}

\section{Study design and population}

This was an open-label single-center prospective randomized explorative study. The study was carried out in accordance with Good Clinical Practice guidelines, the Declaration of Helsinki, and received ethical approval from NRES Committee Yorkshire \& Humber in Leeds East, UK (No. 10/H1306/40).

Following acquisition of informed consent, the study enrolled patients with CKD stages 3 to 5 (estimated glomerular filtration rate of $<60 \mathrm{~mL} / \mathrm{min} / 1.73 \mathrm{~m}^{2}$ ) with a serum ferritin (SF) level less than $200 \mu \mathrm{g} / \mathrm{L}$ and/or a transferrin saturation (TSAT) of $<20 \%$. Further information on the inclusion and exclusion criteria and methodology can be found in the published protocol (Supplementary Table 1, available online) [19].

Patients were recruited and randomly allocated in a 1: 1:1:1 ratio to receive a single infusion of either $200 \mathrm{mg}$ IS (Venofer, Vifor Pharma, Paris, France), 200 mg ID (Cosmofer, Pharmacosmos A/S, Holbaek, Denmark), or $200 \mathrm{mg}$ or 1,000 mg FDI (Monofer, Pharmacosmos A/S) as per summary product characteristics and standard practice in our institution. They were followed up at 2 hours, 1 day, 1 week, and 1 and 3 months after infusion (Supplementary Table 2, available online).

\section{Investigations}

Oxidative stress, labile plasma iron, and inflammatory markers

Thiobarbituric acid reactive substances (TBARS) were measured as a by-product of lipid peroxidation and a surrogate marker of reactive oxygen species generation. The FerOS assay (Afferix, Tel Aviv, Israel) was used to measure LPI. This assay employs a selective chelator that blocks iron redox cycling leading to the identification of iron-mediated reactive oxygen species formation; this measures all forms of non-transferrin bound iron (NTBI), both redox active and not.

Interleukin (IL)-1 $\beta$ IL-6, IL-8, and IL-10 were measured using enzyme-linked immunosorbent assays (ELISA) supplied by Thermo Fisher Scientific (Carlsbad, CA, USA). Beckman Coulter technology (Danaher Corp., Brea, CA, USA) was used for measurement of C-reactive protein (CRP) and albumin. 


\section{Hemoglobin and hematinics}

Hemoglobin was analyzed with Sysmex XN technology (Sysmex UK, Milton Keynes, UK) as per local hospital pathology laboratory practice. SF and TSAT were measured spectrophotometrically using Beckman Coulter analyzers.

\section{Vascular and endothelial function}

The Enverdis Vascular Explorer (Enverdis GmBH Medical Solutions, Jena, Germany) was used to measure pulse wave velocity (PWV) and augmentation index as markers of arterial stiffness. The human CD62P ELISA kit (P-selectin; Abcam, Cambridge, UK) and the mouse E-selectin/CD62E Quantikine ELISA kit (R\&D Systems, Minneapolis, MN, USA) were used to analyze P-selectin and E-selectin, respectively.

\section{Quality of life}

The Short Form (36) Health Survey (SF-36) was used. This is composed of 36 questions calculating patient-reported health status and has been established as a valid and reliable method for the assessment of quality of life in CKDassociated anemia [20].

\section{Safety}

Safety assessment took place throughout the study. Adverse events were recorded and analyzed regarding relationship to treatment with the investigational drug.

\section{Statistical analysis}

This was an explorative pilot study examining proofof-concept; a power calculation was not required. The population used to assess safety included any randomized patient who received any amount of study drug. All analyses involved comparison of outcomes and changes in parameters during the study visits among all participants. Baseline population characteristics have been previously published [19,21] (Supplementary Table 3, 4; available online). The impact of iron treatment for each group individually and for a total combined group was examined for the outcome measures. Descriptive statistics and graphic correlations were employed for the analysis of hematinics, oxidative stress, and inflammatory markers. Numerical data were presented as the mean \pm standard error of the mean. Statistical comparisons of continuous outcomes between the allocated treatment arms were performed using two- way ANOVA (R version 1.2.5019; R Foundation for Statistical Computing, Vienna, Austria). In addition, comparison of high-dose vs. low-dose iron was carried out for the primary and secondary outcome measures. A p-value of $<0.05$ was deemed statistically significant.

\section{Results}

Forty patients, with a mean age of $58.8 \pm 2.2$ years, were recruited and randomized from a total of 49 patients who attended screening. Twenty-three patients $(57.5 \%)$ were male.

One participant commenced hemodialysis before the last visit, but follow-up was completed as per protocol; the patient was included in the analysis. One patient became pregnant within 1 month of receiving 1,000 mg FDI, while another individual randomized to the $1,000 \mathrm{mg}$ FDI group required a second IV iron infusion during the study due to severe symptomatic anemia. The latter two participants were excluded from the 3-month analysis. The flow of participants is summarized in Fig. 1.

\section{Oxidative stress and labile plasma iron}

Administration of IV iron resulted in a rise in mean TBARS level within 2 hours (pre-infusion: 1,083.0 $\pm 117.1 \mathrm{nM}$, 2 hours post-infusion: $1,552.6 \pm 156.0 \mathrm{nM} ; \mathrm{p}=0.060$, all groups combined) (Fig. 2). The increased levels returned to baseline within 1 week. The greatest rise in TBARS was noted in the 1,000 mg FDI group, which was not statistically significant (pre-infusion: $846.0 \pm 108.9 \mathrm{nM}, 2$ hours postinfusion: $1,865.0 \pm 203.2 \mathrm{nM} ; \mathrm{p}=0.250$ ). There was a nonstatistically significant increase with IS that occurred 1 week post-infusion (pre-infusion: $906.3 \pm 140.9 \mathrm{nM}, 1$ week postinfusion: $1,261.3 \pm 369.3 \mathrm{nM} ; \mathrm{p}=0.990$ ). There were no statistically significant differences for the effect on TBARS between products used or between the high-dose and lowdose FDI.

Mean LPI levels increased significantly within 2 hours of infusion and returned to baseline within 1 week (preinfusion: $1.4 \pm 0.5 \Delta \mathrm{FU} / \mathrm{min}, 2$ hours post-infusion: $7.4 \pm$ 2.4 $\Delta \mathrm{FU} / \mathrm{min} ; \mathrm{p}=0.006$, all groups combined) (Fig. 3). LPI increased in the ID and IS groups, but these did not reach statistical significance. The concentration of LPI with 200 mg FDI remained constant and similar to the baseline level 
Invited to participate and attended screening $(n=49)$

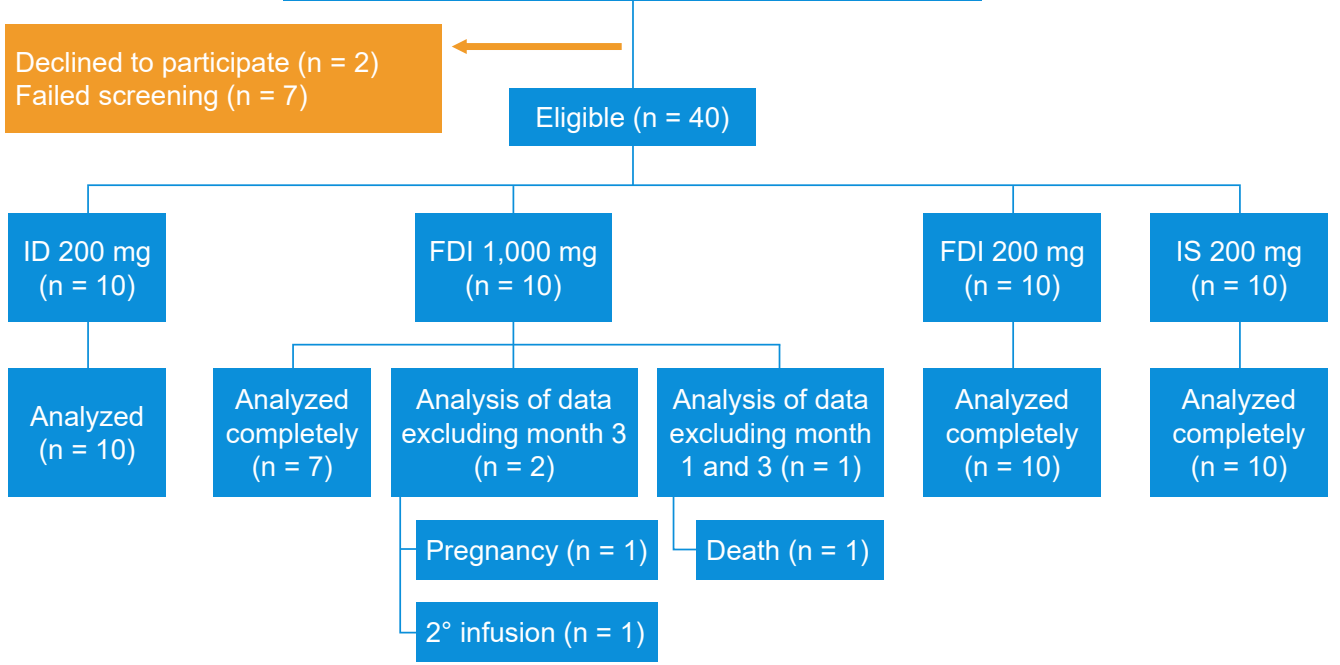

Figure 1. Consolidated Standards Of Reporting Trials (CONSORT) diagram. It indicates the flow of patients from point of identification to enrollment in the trial and also displays the patients who were included in the statistical analysis.

FDI, ferric derisomaltose; ID, iron dextran; IS, iron sucrose.

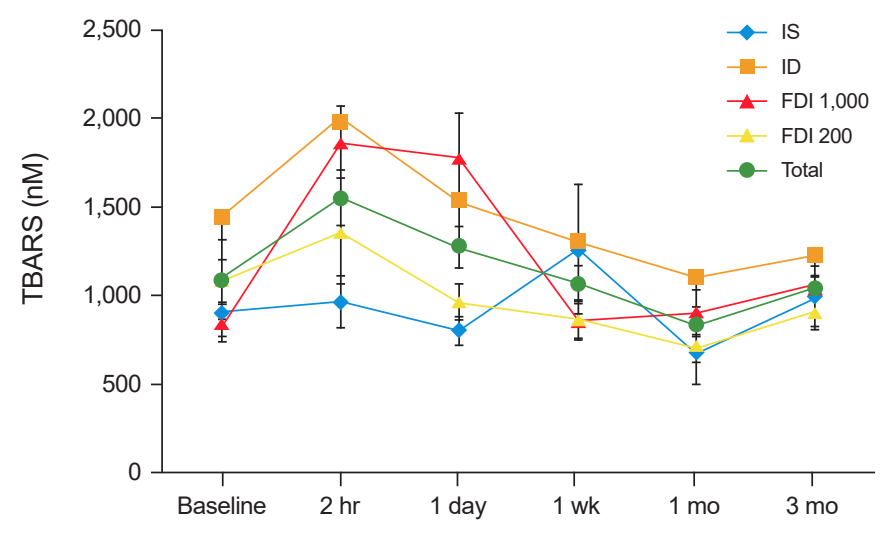

Figure 2. Comparative effects on oxidative stress. Thiobarbituric acid reactive substances (TBARS) measurements are plotted as means with error bars representing standard error of the mean for all individual groups and for a total combined group. FDI, ferric derisomaltose; ID, iron dextran; IS, iron sucrose.

throughout the study. There was a significant increase in LPI with 1,000 mg FDI (pre-infusion: $0.33 \pm 0.2 \Delta \mathrm{FU} / \mathrm{min}, 2$ hours post-infusion: $19.6 \pm 7.1 \Delta \mathrm{FU} / \mathrm{min} ; \mathrm{p}<0.001$ ), and the level at 2 hours post-infusion was significantly higher when compared to the $200 \mathrm{mg}$ FDI group $(19.6 \pm 7.1 \Delta \mathrm{FU} / \mathrm{min}$ vs. $1.6 \pm 0.8 \Delta \mathrm{FU} / \mathrm{min} ; \mathrm{p}<0.001)$. These changes resolved within 1 week.

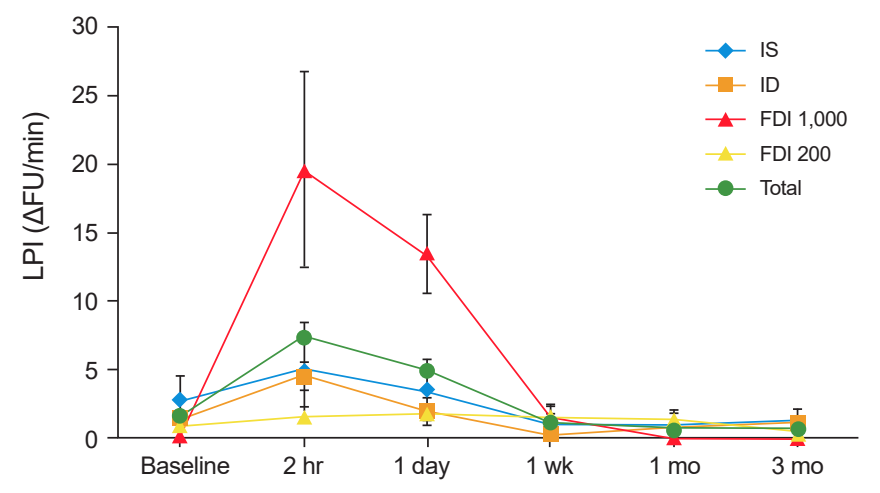

Figure 3. Comparative effects on labile plasma iron (LPI). LPI measurements are plotted as mean values for all groups individually and for a total combined group. Mean values are plotted and the error bars represent standard error of the mean. FDI, ferric derisomaltose; ID, iron dextran; IS, iron sucrose.

\section{Inflammation}

There was a rise in mean CRP level within a day of infusion, which returned to baseline levels within 1 month (preinfusion: $7.5 \pm 1.6 \mathrm{mg} / \mathrm{L}, 1$ day post-infusion: $17.6 \pm 8.0 \mathrm{mg} /$ $\mathrm{L} ; \mathrm{p}=0.400 / 1$ month post-infusion: $7.5 \pm 1.7 \mathrm{mg} / \mathrm{L} ; \mathrm{p}>0.999$, all groups combined). This rise was more evident in patients receiving IS (pre-infusion: $8.1 \pm 3.3 \mathrm{mg} / \mathrm{L}, 1$ day post-infusion: $36.1 \pm 27.0 \mathrm{mg} / \mathrm{L} ; \mathrm{p}=0.550$ ). The changes in CRP did not 
reach statistical significance for FDI at any dose (Fig. 4).

A transient fall in IL-10 within one month and a rise in IL-8 within 1 week of IV iron infusion were observed across the treatment groups; IL-6 was unaffected. IL- $1 \beta$ did not reach detectable levels during the study. A transient rise in IL-10 within 2 hours of infusion was noted with IS (Supplementary Fig. 1, 2; available online).

Serum albumin concentration remained unchanged throughout the study for all IVIPs (Supplementary Fig. 3, available online).

\section{Hemoglobin and hematinics}

For all groups combined, hemoglobin concentration rose to its maximal level after one month and was sustained until the end of the study at 3 months ( $p>0.999)$. The increase in hemoglobin concentration was not significantly different between the iron compounds, and no statistically significant

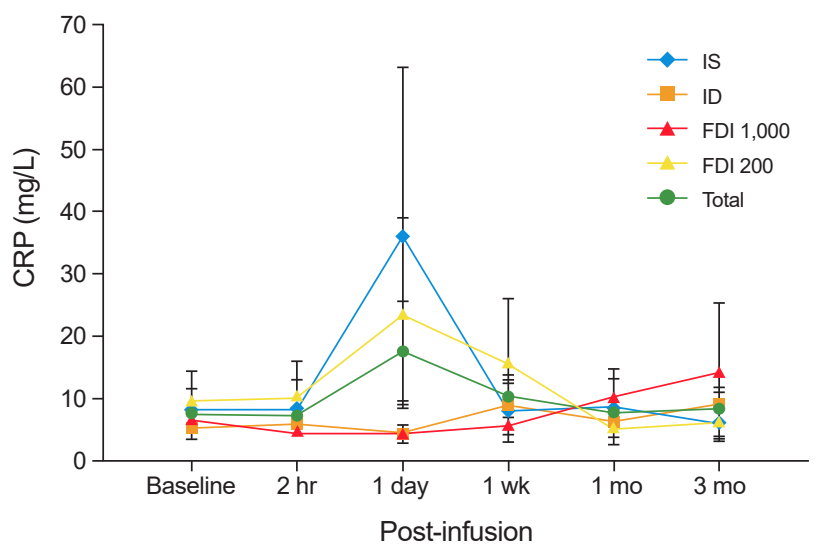

Figure 4. Comparative effects on CRP. CRP measurements plotted as means with error bars representing standard error of the mean for each individual group and for a total combined group.

FDI, ferric derisomaltose; ID, iron dextran; IS, iron sucrose. difference was noted between high-dose and low-dose FDI ( $p>0.999$ throughout study) (Table 1).

TSAT increased within 2 hours post-infusion (up to $80 \%$, all groups combined) and returned to baseline level by the first week (Fig. 5). This transient rise from baseline to 2 hours post-infusion was statistically significant in both the 1,000 mg FDI $(17.8 \%$ to $98.7 \%$; $\mathrm{p}<0.001)$ and IS groups $(21.1 \%$ to $91.4 \%$; $\mathrm{p}<0.001)$. High-dose FDI produced a significant change in TSAT at 2 hours and 1 day post-infusion when compared with low-dose FDI that persisted for 1 week (FDI of 1,000 mg vs. $200 \mathrm{mg}$; 2 hours post-infusion: $98.7 \%$ vs. $58.3 \%, \mathrm{p}=0.005$; 1 day post-infusion: $100 \%$ vs. $51.8 \%$, $\mathrm{p}<0.001$ ). There was no statistically significant difference between the different iron preparations at 2 hours postinfusion.

The mean SF level rose within 2 hours post-infusion to achieve its maximal mean concentration at 1 week (preinfusion: $68.8 \pm 8.0 \mu \mathrm{g} / \mathrm{L}$, 1 week post-infusion: $216.2 \pm 36.6$ $\mu \mathrm{g} / \mathrm{L}, 3$ months post-infusion: $122.6 \pm 23.1 \mu \mathrm{g} / \mathrm{L}$; all groups

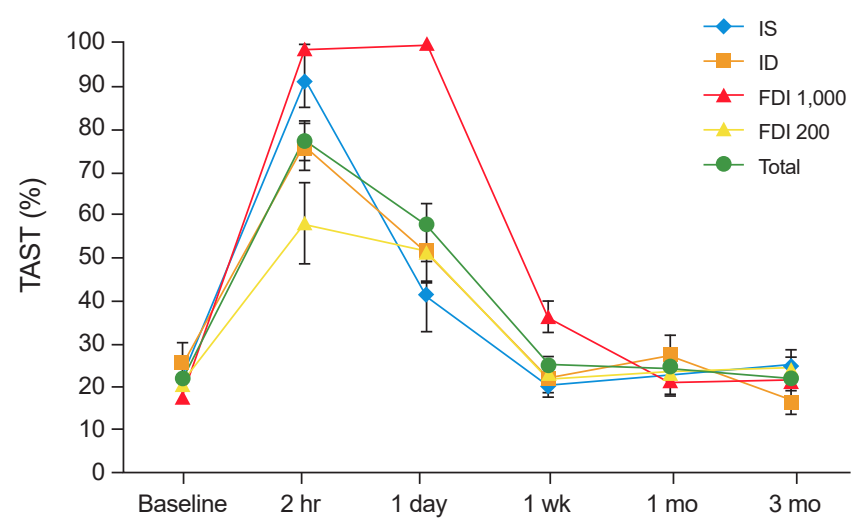

Figure 5. Comparative effects on transferrin saturation (TSAT). TSAT measurements are plotted as a mean percentage for each individual group and for a total combined group. Mean values are plotted with error bars representing standard error of the mean. FDI, ferric derisomaltose; ID, iron dextran; IS, iron sucrose.

Table 1. Analysis of hemoglobin concentration

\begin{tabular}{|c|c|c|c|c|c|c|}
\hline \multirow{2}{*}{ Concentration (g/L) } & \multirow{2}{*}{ Baseline } & \multicolumn{5}{|c|}{ Post-infusion } \\
\hline & & $2 \mathrm{Hr}$ & 1 Day & $1 \mathrm{Wk}$ & $1 \mathrm{Mo}$ & $3 \mathrm{Mo}$ \\
\hline Iron sucrose, 200 mg & $122.1 \pm 4.6$ & $125.6 \pm 5.3$ & $120.1 \pm 4.6$ & $125.4 \pm 5.3$ & $130.7 \pm 5.9$ & $129.0 \pm 7.3$ \\
\hline Iron dextran, 200 mg & $129.6 \pm 7.5$ & $127.0 \pm 7.5$ & $124.9 \pm 8.1$ & $126.4 \pm 7.4$ & $127.8 \pm 6.2$ & $130.9 \pm 7.4$ \\
\hline Ferric derisomaltose, $1,000 \mathrm{mg}$ & $115.5 \pm 4.5$ & $117.8 \pm 4.7$ & $116.5 \pm 4.5$ & $114.8 \pm 5.8$ & $113.6 \pm 5.5$ & $120.0 \pm 8.8$ \\
\hline Ferric derisomaltose, 200 mg & $123.0 \pm 5.5$ & $125.9 \pm 5.8$ & $122.3 \pm 6.1$ & $123.1 \pm 6.2$ & $126.0 \pm 5.8$ & $126.0 \pm 6.1$ \\
\hline Total & $122.6 \pm 2.8$ & $123.7 \pm 2.9$ & $120.7 \pm 2.9$ & $122.7 \pm 3.1$ & $124.6 \pm 3.0$ & $127.2 \pm 3.6$ \\
\hline
\end{tabular}

Data are presented as mean \pm standard error of the mean. 


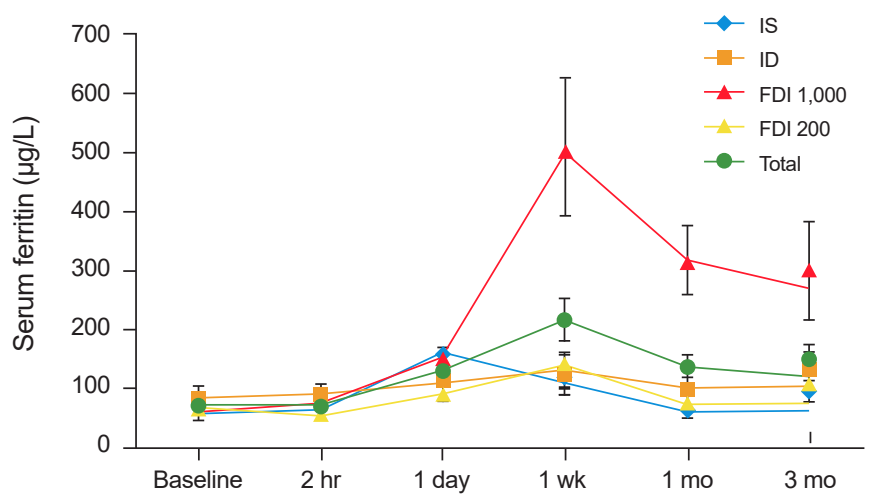

Figure 6. Comparative effects on serum ferritin. Serum ferritin measurements are plotted as means with error bars representing standard error of the mean for each individual group and for a total combined group.

FDI, ferric derisomaltose; ID, iron dextran; IS, iron sucrose.

combined) (Fig. 6). The 1,000 mg FDI group produced the greatest and longest-lasting iron repletion (pre-infusion: $69.1 \pm 18.4 \mu \mathrm{g} / \mathrm{L}$, 1 week post-infusion: $505.9 \pm 105.5 \mu \mathrm{g} / \mathrm{L}, 3$ months post-infusion: $271.0 \pm 83.3 \mu \mathrm{g} / \mathrm{L}$ ), which remained significantly higher than baseline throughout the study (baseline to 1 week post-infusion, $\mathrm{p}<0.001$; baseline to 3 months post-infusion, $\mathrm{p}=0.007$ ). The $1,000 \mathrm{mg}$ FDI dose achieved a statistically significantly greater change in SF when compared to the $200 \mathrm{mg}$ FDI dose throughout the study $(\mathrm{p}<0.001)$.

\section{Vascular and endovascular function}

There was a trend for a reduction in mean PWV throughout the study across all groups, which did not reach statistical significance (pre-infusion: $7.5 \pm 0.4 \mathrm{~m} / \mathrm{sec}, 3$ months postinfusion: $6.7 \pm 0.4 \mathrm{~m} / \mathrm{sec} ; \mathrm{p}>0.999$ ). There was no significant difference between the compound used and improvement in PWV. No difference was noted between high-dose and low-dose FDI. A similar improvement tendency was observed for augmentation index (Supplementary Table 5, available online).

IV iron did not significantly affect E-selectin during the study, regardless of iron preparation or dose. A nonstatistically significant decrease in mean P-selectin level was seen (pre-infusion: $75.0 \pm 6.4 \mathrm{ng} / \mathrm{mL}$; 2 hours post-infusion: $72.4 \pm 6.5 \mathrm{ng} / \mathrm{mL} ; 3$ months post-infusion: $68.7 \pm 6.9 \mathrm{ng} / \mathrm{mL}$; $\mathrm{p}>0.999$, all groups combined); no significant differences in P-selectin were seen between the different IV iron groups or between different doses of FDI (Supplementary Table 5).

\section{Quality of Life}

There was a trend for improvement in all domains of the SF36 following iron administration for all iron treatment groups combined and with each compound separately through the completion of the study. This was not statistically significant (Supplementary Table 6, available online).

\section{Safety}

One death occurred during the study due to myocardial infarction in a patient with significant cardiovascular comorbidities, 1 week following infusion of iron $(1,000$ mg FDI). The event was deemed unrelated to the study drug. There were no episodes of anaphylaxis or infusion reactions. No infection-related hospitalizations and no other cardiovascular events occurred.

\section{Discussion}

In this IRON-CKD study, we examined whether administration of IVIPs with different structural stability and risk of labile iron release would have differential impacts on biomarkers of oxidative stress and inflammation and on measures of efficacy, endothelial function, and safety in iron-deficient individuals with CKD. We also compared different doses of FDI, a commonly used third-generation IVIP with low risk of labile release, to identify any dosage-related differences.

Previously, it was assumed that a short-lived increase in oxidative stress was pathogenic. In this study, markers of oxidative stress returned to baseline within 1 week irrespective of iron preparation and dose. Here, despite a trend for increase in TBARS with high-dose FDI and IS, this did not reach statistical significance at any point. LPI rose in a dose-dependent manner, with a statistically significant rise noted with the high-dose FDI. Indeed, results from cellular studies with IVIP have commented on ironinduced increases in cellular stress that are possibly doseand tissue-dependent; however, no duration of effect was evaluated [22]. The dose-dependent rise displayed here was short-lived and did not translate into systemic adverse effects nor a statistically significant increase in oxidative stress and inflammatory markers. The unchanged LPI from 
baseline with $200 \mathrm{mg}$ FDI potentially reflects the controlled iron delivery to iron-binding proteins with FDI. This results from the tight binding of iron in the carbohydrate matrix of FDI, which translates into a highly stable compound when compared to second-generation iron preparations such as IS [23]. Garbowski et al. [24] recently commented on two iron release modes; early (direct delivery of iron to transferrin in blood) and late (indirect release of iron from macrophages), with differences between IV irons dependent on their pharmacokinetic properties. They also concluded that FDI had a lower NTBI level compared to IS [24], similar to the findings in the current IRON-CKD study showing lower levels of NTBI with FDI vs. IS and ID at equivalent doses. The lack of impact on oxidative stress by FDI could explain the recent findings of the FERWON-NEPHRO trial where high-dose FDI (1,000 mg), when compared to IS ( $5 \times$ $200 \mathrm{mg}$ over 2 weeks), resulted in an improved incidence of cardiovascular events [25].

This can be inferred further through the transient rise in CRP with IS, a compound with weaker iron binding. The brief rise in IL-10 (an anti-inflammatory cytokine) caused by IS could represent part of a regulatory process in response to IL-12 (a pro-inflammatory cytokine) [26]. The lack of significant effect on inflammatory markers with third-generation IV iron, even at a high dose (FDI of 1,000 $\mathrm{mg}$ ), may indicate that no LPI toxicity occurs with such compounds.

The rise in TSAT within hours in all groups demonstrates that current available iron preparations are efficacious, delivering iron rapidly and providing a source of circulating iron. SF achieved maximal rise after one week with 1,000 mg FDI, which provided the most significant and lasting elevation. This may reflect a greater iron repletion effect and highlights that a higher dose of IV iron is able to produce substantial and more effective iron repletion. It is important to underline that at maximal SF concentrations, achieved with high-dose FDI, there was no induced oxidative stress. These results reveal greater iron replenishment using high doses in a manner similar to the landmark PIVOTAL (Proactive IV Iron Therapy in Hemodialysis Patients) study [27]. The modest increase in hemoglobin is consistent with previous studies in non-anemic patients demonstrating little change [28].

IV iron did not aggravate arterial stiffness. This is consistent with other trials in patients receiving dialysis where no change in vascular reactivity was noted [29]. Here, treatment with IV iron resulted in a tendency for improvement in PWV and augmentation index.

Upregulation of selectins is associated with atherosclerosis and inflammation [30]. Evidence on the impact of iron on cell adhesion molecules such as E-selectin and P-selectin is scarce and conflicting [31,32]. In the current study, there were no significant changes in the expression of either E-selectin or P-selectin with any of the IVIPs or doses, suggesting that IV iron does not affect selectin-driven pathways.

This study has several limitations. It was open-label, which increases the risk of observer and participant bias. The study focused on non-smokers and may therefore not be representative of potential interactions of smoking (a powerful pro-oxidant factor) with IV iron treatment; however, it does not exclude any external effect of smoking. Moreover, the small number of participants in each of the four groups did not allow for stratification according to age and comorbidities, renal function, and medications, which are potentially important factors affecting oxidative stress. Additionally, as a pilot study it was not statistically powered for definitive conclusions; however, it can be used for hypothesis generation and for trend observation.

IRON-CKD provided a direct comparative assessment of the relative acute treatment effects of commonly used IV irons. The results indicate that, in the short term and following a single IV iron infusion, endothelial function and oxidative status are not affected. All studied preparations led to a rise in both stored and circulating iron, with more effective repletion with a higher dose. This translated into a small improvement in quality of life, in line with previous large cohort trials [4] underlining the impact of iron in nonanemic patients as witnessed in inflammatory bowel disease [33]. There were no infection-related hospital admissions with any of the preparations administered or with either low- or high-dose IV iron, which supports the good safety profile of high-dose iron and complements the findings in the PIVOTAL study where infection rates were identical irrespective of high- or low-dose IV iron treatment [34].

Additionally, this IRON-CKD study assessed the dosedependent effects of FDI, supplementing evidence on its efficacy from studies in CKD patients comparing FDI with oral iron and second-generation IVIP [35,36]. As third-generation IV iron compounds share different 
pharmacodynamic properties from their predecessors [23], previous results on dose-associated toxicity cannot be necessarily extrapolated as indicated by this study.

Nuhu et al. [37] recently examined the mechanistic links between iron and CKD and concluded that the overall oxidation status in CKD is antioxidant. An interplay exists between iron homeostasis and oxidative stress in CKD that in turn impacts the effects of IV iron treatment [38]. It is believed that in CKD the system is primed with activation of both pro- and antioxidant pathways; iron deficiency may aggravate oxidative stress. It is, therefore, speculated that the resolution of iron deficiency with IV iron (despite a short transient increase in oxidative stress) provides an overall net antioxidant benefit due to mobilization of relevant enzymes [39].

The impact of multiple iron infusions and the long-term safety profile of IV iron require further research. Additional exploration of the impact of third-generation IVIP at comparative doses is needed, given their pharmacochemical differences and the potential impact on bone metabolism due to hypophosphatemia as reported with ferric carboxymaltose [40]. Such mechanistic evidence should be considered when incorporating IV iron therapy into other areas of medicine.

Although the patient sample size is too small to make any firm conclusions, this study provides evidence that currently available IV iron therapies are efficacious with a good safety profile. Additionally, this study revealed that high-dose FDI displays a trend of a greater treatment effect, resulting in higher SF and TSAT compared to a lower dose of FDI and to other IV iron preparations. This does not negatively affect oxidative stress, endothelial function, or inflammation. The successful IV iron repletion translates to improved quality of life and may positively affect arterial stiffness. Further larger studies are required to confirm the potential oxidative effects of IV iron and the impact of multiple doses in this patient group.

\section{Conflicts of interest}

The results presented in this paper have not been published previously in whole or part, except in abstract format following the ERA/EDTA-2020 Virtual Conference. Sunil Bhandari has received honorarium, consultancy fees, membership advisory board, and travel funding from
Pharmacosmos A/S, Vifor Pharma, and Astellas. The rest of the authors have no conflicts of interest.

\section{Funding}

The IRON-CKD study received an unrestricted grant from Pharmacosmos A/S. The funder had no role in the study design, data collection and analysis, decision to publish, or preparation of the manuscript.

\section{Acknowledgments}

The authors would like to thank Tracy Cathcart, Vikki Jubb, Ahmed Ziedan, and the Research and Development Team (Leanne Cox and Sarah Moffat) at the Hull University Teaching Hospitals NHS Trust for their support.

\section{Authors' contributions}

Conceptualization: SB, RS

Data curation: XK

Formal analysis: $\mathrm{XK}$

Funding acquisition: SB

Investigation: $\mathrm{SB}$

Methodology: SB, AG, RS

Project administration: SB, AG

Visualisation: XK

Writing-original draft: $\mathrm{XK}$

Writing-review \& editing: XK, SB, AG, RS

All authors read and approved the final manuscript.

\section{Data statement}

Individual participant data that underlie the results reported in this article, after de-identification (text, tables, figures, and appendices), alongside protocols are available on request for 3 years following online publication to researchers with a methodologically sound proposal for the potential inclusion in meta-analysis. Requests should be directed to xenophon. kassianides@hey.nhs.uk.

\section{ORCID}

Xenophon Kassianides, https://orcid.org/0000-0002-1452-7757

Andrew Gordon, https://orcid.org/0000-0003-2770-6129 
Roger Sturmey, https://orcid.org/0000-0003-4423-5058
Sunil Bhandari, https://orcid.org/0000-0002-0996-9622

\section{References}

1. GBD 2016 Disease and Injury Incidence and Prevalence Collaborators. Global, regional, and national incidence, prevalence, and years lived with disability for 328 diseases and injuries for 195 countries, 1990-2016: a systematic analysis for the Global Burden of Disease Study 2016. Lancet 2017;390:12111259.

2. Camaschella C. Iron-deficiency anemia. N Engl J Med 2015;372: 1832-1843.

3. Keshav S, Stevens R. New concepts in iron deficiency anaemia. Br J Gen Pract 2017;67:10-11.

4. Bhandari S, Pereira DIA, Chappell HF, Drakesmith H. Intravenous irons: from basic science to clinical practice. Pharmaceuticals (Basel) 2018;11:82.

5. National Institute for Health and Care Excellence. NICE guideline NG8. Chronic kidney disease: managing anaemia [Internet]. London (UK): National Institute for Health and Care Excellence, c2015 [cited 2020 Jul 7]. Available from: https://www.nice.org. uk/guidance/ng8.

6. Kidney Disease: Improving Global Outcomes (KDIGO) Anemia Work Group. KDIGO clinical practice guideline for anemia in chronic kidney disease. Kidney Int Suppl 2012;2:279-335.

7. Agarwal R, Kusek JW, Pappas MK. A randomized trial of intravenous and oral iron in chronic kidney disease. Kidney Int 2015;88:905-914.

8. Zager RA, Johnson AC, Hanson SY. Parenteral iron nephrotoxicity: potential mechanisms and consequences. Kidney Int 2004; 66:144-156.

9. Mimić-Oka J, Savić-Radojević A, Pljesa-Ercegovac M, et al. Evaluation of oxidative stress after repeated intravenous iron supplementation. Ren Fail 2005;27:345-351.

10. Shah SV, Baliga R, Rajapurkar M, Fonseca VA. Oxidants in chronic kidney disease. J Am Soc Nephrol 2007;18:16-28.

11. Cabantchik ZI. Labile iron in cells and body fluids: physiology, pathology, and pharmacology. Front Pharmacol 2014;5:45.

12. Bresgen N, Eckl PM. Oxidative stress and the homeodynamics of iron metabolism. Biomolecules 2015;5:808-847.

13. Zager RA, Johnson AC, Hanson SY, Wasse H. Parenteral iron formulations: a comparative toxicologic analysis and mechanisms of cell injury. Am J Kidney Dis 2002;40:90-103.

14. Johnson AC, Becker K, Zager RA. Parenteral iron formulations differentially affect MCP-1, HO-1, and NGAL gene expression and renal responses to injury. Am J Physiol Renal Physiol 2010; 299:F426-F435.

15. Jahn MR, Andreasen HB, Fütterer S, et al. A comparative study of the physicochemical properties of iron isomaltoside 1000 (Monofer), a new intravenous iron preparation and its clinical implications. Eur J Pharm Biopharm 2011;78:480-491.

16. Zheng H, Huang X, Zhang Q, Katz SD. Iron sucrose augments homocysteine-induced endothelial dysfunction in normal subjects. Kidney Int 2006;69:679-684.

17. Kuo KL, Hung SC, Lee TS, Tarng DC. Iron sucrose accelerates early atherogenesis by increasing superoxide production and upregulating adhesion molecules in CKD. J Am Soc Nephrol 2014;25:2596-2606.

18. Agarwal R, Rizkala AR, Kaskas MO, Minasian R, Trout JR. Iron sucrose causes greater proteinuria than ferric gluconate in nondialysis chronic kidney disease. Kidney Int 2007;72:638-642.

19. Ziedan A, Bhandari S. Protocol and baseline data for a prospective open-label explorative randomized single-center comparative study to determine the effects of various intravenous iron preparations on markers of oxidative stress and kidney injury in chronic kidney disease (IRON-CKD). Trials 2019;20:194.

20. Finkelstein FO, van Nooten F, Wiklund I, Trundell D, Cella D. Measurement properties of the Short Form-36 (SF-36) and the Functional Assessment of Cancer Therapy-Anemia (FACT-An) in patients with anemia associated with chronic kidney disease. Health Qual Life Outcomes 2018;16:111.

21. Ziedan A, Kassianides X, Bhandari S. Correction to: protocol and baseline data for a prospective open-label explorative randomized singlecenter comparative study to determine the effects of various intravenous iron preparations on markers of oxidative stress and kidney injury in chronic kidney disease (IRON-CKD). Trials 2020;21:273.

22. Connor JR, Zhang X, Nixon AM, Webb B, Perno JR. Comparative evaluation of nephrotoxicity and management by macrophages of intravenous pharmaceutical iron formulations. PLoS One 2015;10:e0125272

23. Fütterer S, Andrusenko I, Kolb U, Hofmeister W, Langguth P. Structural characterization of iron oxide/hydroxide nanoparticles in nine different parenteral drugs for the treatment of iron deficiency anaemia by electron diffraction (ED) and X-ray powder diffraction (XRPD). J Pharm Biomed Anal 2013;86:151-160.

24. Garbowski MW, Bansal SS, Porter JB, Burckhardt S, Hider R. NTBI is transiently generated from two compartments during a single dose of intravenous iron - a randomized controlled 
clinical study. Blood 2019;134(Suppl 1):2234.

25. Bhandari S, Kalra PA, Berkowitz M, Belo D, Thomsen LL, Wolf M. Safety and efficacy of iron isomaltoside 1000/ferric derisomaltose versus iron sucrose in patients with chronic kidney disease: the FERWON-NEPHRO randomized, openlabel, comparative trial. Nephrol Dial Transplant 2020 Feb 12 [Epub]. Doi: 10.1093/ndt/gfaa011.

26. Ma X, Yan W, Zheng H, et al. Regulation of IL-10 and IL-12 production and function in macrophages and dendritic cells. F1000Res 2015;4:F1000 Faculty Rev-1465.

27. Macdougall IC, White C, Anker SD, et al. Intravenous iron in patients undergoing maintenance hemodialysis. $N$ Engl J Med 2019;380:447-458.

28. Miles LF, Litton E, Imberger G, Story D. Intravenous iron therapy for non-anaemic, iron-deficient adults. Cochrane Database Syst Rev 2019;12:CD013084.

29. Schaller G, Scheiber-Mojdehkar B, Wolzt M, et al. Intravenous iron increases labile serum iron but does not impair forearm blood flow reactivity in dialysis patients. Kidney Int 2005;68:2814-2822.

30. McEver RP. Selectins: initiators of leucocyte adhesion and signalling at the vascular wall. Cardiovasc Res 2015;107:331-339.

31. Kartikasari AE, Georgiou NA, Visseren FL, van Kats-Renaud $\mathrm{H}$, van Asbeck BS, Marx JJ. Intracellular labile iron modulates adhesion of human monocytes to human endothelial cells. Arterioscler Thromb Vasc Biol 2004;24:2257-2262.

32. Kulnigg-Dabsch S, Schmid W, Howaldt S, et al. Iron deficiency generates secondary thrombocytosis and platelet activation in IBD: the randomized, controlled thromboVIT trial. Inflamm Bowel Dis 2013;19:1609-1616.

33. Eliadou E, Kini G, Huang J, Champion A, Inns SJ. Intravenous iron replacement improves quality of life in hypoferritinemic inflammatory bowel disease patients with and without anemia. Dig Dis 2017;35:444-448.

34. Macdougall IC, Bhandari S, White C, et al. Intravenous iron dosing and infection risk in patients on hemodialysis: a prespecified secondary analysis of the PIVOTAL trial. J Am Soc Nephrol 2020;31:1118-1127.

35. Bhandari S, Kalra PA, Kothari J, et al. A randomized, openlabel trial of iron isomaltoside 1000 (Monofer ${ }^{\circledR}$ ) compared with iron sucrose (Venofer ${ }^{\circledR}$ ) as maintenance therapy in haemodialysis patients. Nephrol Dial Transplant 2015;30:1577-1589.

36. Kalra PA, Bhandari S, Saxena S, et al. A randomized trial of iron isomaltoside 1000 versus oral iron in non-dialysisdependent chronic kidney disease patients with anaemia. Nephrol Dial Transplant 2016;31:646-655.

37. Nuhu F, Seymour AM, Bhandari S. Impact of intravenous iron on oxidative stress and mitochondrial function in experimental chronic kidney disease. Antioxidants (Basel) 2019;8:498.

38. Koskenkorva-Frank TS, Weiss G, Koppenol WH, Burckhardt S. The complex interplay of iron metabolism, reactive oxygen species, and reactive nitrogen species: insights into the potential of various iron therapies to induce oxidative and nitrosative stress. Free Radic Biol Med 2013;65:1174-1194.

39. Meroño T, Dauteuille C, Tetzlaff W, et al. Oxidative stress, HDL functionality and effects of intravenous iron administration in women with iron deficiency anemia. Clin Nutr 2017;36:552-558.

40. Wolf M, Rubin J, Achebe M, et al. Effects of iron isomaltoside vs ferric carboxymaltose on hypophosphatemia in iron-deficiency anemia: two randomized clinical trials. JAMA 2020;323:432-443. 